\title{
Synergy Between Novel Antimicrobials and Conventional Antibiotics or Bacteriocins
}

\author{
KRYSTYNA I. WOLSKA*, KATARZYNA GRZEŚ and ANNA KUREK
}

Department of Bacterial Genetics, Institute of Microbiology, Faculty of Biology,

University of Warsaw, Warsaw, Poland

Received 9 November 2011, revised 7 April 2012, accepted 7 May 2012

\author{
Abstract
}

\begin{abstract}
Due to the alarming spread of resistance to classic antimicrobial agents, innovative therapeutic methods to combat antibiotic-resistant bacterial pathogens are urgently required. This minireview examines the enhancement of antibiotic efficacy by their combination with new antimicrobials, such as plant-derived compounds, metal ions and nanoparticles and bacteriophage lytic enzymes. The mechanisms of the observed synergy are also described. The promising results of basic research indicate that in future, combined therapy may be applied in human and veterinary medicine, agriculture and the food industry to combat bacterial pathogens.
\end{abstract}

Ke y words: antibiotics, bacteriophages, nanoparticles, plant compounds, synergy

\section{Introduction}

The extensive use of antibiotics has led to growing resistance and the spread of many bacterial pathogens, which now constitutes a serious medical problem. For this reason, the number of studies aimed at developing new analogs of known antibiotics, e.g. oxazolidinones, glycopeptides, quinolones, aminoglycosides, tetracyclines and ketolides (Theuretzbacher, 2011), and at identifying novel antibacterial therapeutics and strategies, is growing exponentially. Positive validation of new antimicrobial agents is often connected with the discovery of novel targets. To illustrate this point, the so-called switch region of bacterial RNA polymerase, that does not overlap the rifamycin binding site, has been confirmed as the target of mycopyronin, corallopyronin, ripostatin and lipiarmycin - antibiotics which are not cross-resistant with rifamycins (Srivastava et al., 2011). It was also recently postulated that NF- $\kappa B$ (nuclear transcription factor- $\kappa B$ ), which is crucial for the cellular response to stress and inflammation caused by e.g. microbial infection, could represent a target for antimicrobial and antiviral therapies (Vitiello et al., 2012). In addition, there is current controversy over whether fatty acid biosynthesis pathways may constitute a novel promising target for antibiotics to control bacterial pathogens, particu- larly Staphylococcus aureus (Parson and Rock, 2011). Potential antibacterial treatments include the use of antimicrobial peptides, antivirulence strategies and therapeutic antibodies (Fernebro, 2011), as well as plantderived compounds, metal nanoparticles and bacteriophage lytic enzymes.

Plant-derived compounds, metal nanoparticles and bacteriophage lysins, which are the subject of this review, may be considered new antimicrobials due to their proven and substantial antibacterial effect, which is, however, weaker than that of common antibiotics produced by bacteria and fungi (Hemaiswarya et al., 2008). In the last decade, the first steps in elucidating the mechanisms of antibacterial activity and the cellular targets of plant-derived compounds have been made, with phenolics, and especially flavones, being the subject of the majority of studies. Flavones cause disruption of the bacterial cytoplasmic membrane and inhibit energy metabolism (Tsuchiya and Iinuma, 2000; Plaper et al., 2003). These compounds can also attenuate the pathogenicity of various bacteria by their ability to inhibit quorum-sensing signal receptors, sortase and urease activity, listeriolysin $\mathrm{O}$, coagulase and $\alpha$-toxin secretion, and by neutralizing bacterial toxins (for review see Cushnie and Lamb, 2011). There have also been a considerable number of reports describing the antibacterial effect of another group of plant-derived

* Corresponding author: K.I. Wolska, Department of Bacterial Genetics, Institute of Microbiology, University of Warsaw, Miecznikowa 1, 02-096 Warsaw, Poland; phone: (+48) 225541 402; fax: (+48) 225541 402; e-mail: izabelaw@biol.uw.edu.pl 
compounds, the terpenes, but only a few discuss the molecular basis of their antibacterial activity (for review see Kurek et al., 2011; Wolska et al., 2010). For example, pentacyclic triterpenoids can inhibit insoluble glycan synthesis by Streptococcus mutans (Kozai et al., 1999) and peptidoglycan metabolism in Listeria monocytogenes (Kurek et al., 2010), and also influence Escherichia coli gene expression and biofilm formation (Ren et al., 2005; Grudniak et al., 2011). It was also shown that diterpenoids have potent anti-biofilm activity against staphylococci (Walencka et al., 2007). In addition, the sesquiterpene farnesol was found to inhibit S. aureus growth by affecting the mevalonate pathway of isoprenoid synthesis (Kaneko et al., 2011).

Metal ions and nanoparticles, especially silver compounds, constitute another group of potent antimicrobials that have already been applied in medicine and pharmacology (Chopra, 2007; Li et al., 2006; Monteiro et al., 2009). They are used as antibacterial surface coatings on medical devices, such as venous and urinary catheters, implants and megaprostheses (Hamill et al., 2007; Hardes et al., 2010). Silver nanoparticles (AgNPs) display remarkable antimicrobial activity against Pseudomonas aeruginosa, E. coli and Enterobacter cloacae, being more active against Gram-negative than against Gram-positive bacteria (Im et al., 2011). Metal ions and nanoparticles are safer in vitro and have a greater antibacterial effect when stabilized by various polymer surfactants (Lin et al., 2012). Silver nanoparticles can disrupt bacterial membranes (Singh et al., 2008), cause membrane lipid peroxidation (Neal, 2008), alter gene expression (Lok et al., 2006), and when inside the cell, they may also damage DNA and impair the respiratory chain and cell division (Rai et al., 2009).

The lytic enzymes of bacteriophages may also be considered as novel antimicrobials. Bacteriophage therapy to treat bacterial infections has been well studied, but the extensive literature on this subject will not be considered here (for review see Chibani-Chennoufi et al., 2004; Górski et al., 2009). An alternative to whole phage particles is the application of phage lysins: enzymes that are active against Gram-positive bacteria. It was shown that the lytic enzyme PhyPH is active against Bacillus anthracis strains (Yoong et al., 2006), and the S. aureus bacteriophage $\phi M R 11$ lysin, designated MV-L, can inhibit a number of S. aureus strains, including those that are methicillin-resistant (MRSA) or vancomycin-intermediate (VISA) (Rashel et al., 2007). Using a mouse model, Grandgigard and coworkers (2008) demonstrated that the recombinant phage lysine Clp-1 may be useful in therapy for pneumococcal meningitis, and Nelson et al. (2001) found that the lysin isolated from a virulent $C_{1}$ phage, specific for group $\mathrm{C}$ streptococci, can prevent and eliminate upper respiratory tract colonization by group A streptococci.
The compounds listed above display antibacterial activity when used alone, but there is also the possibility of using them in combination with conventional antibiotics in order to improve their efficacy. Combination therapy, i.e. the simultaneous treatment of infections with more than one drug, may constitute an efficient strategy to combat antibacterial resistance. This minireview summarizes current data on the synergistic activity of antibiotics in combination with plantderived compounds, metal ions and nanoparticles, and bacteriophage lytic enzymes. The examples of synergy between novel antimicrobials and antibiotics / bacteriocins are also listed in Table I. The determination of synergy between two compounds is based on calculation of the FICI (fractional inhibitory concentration index), where a value of $\leq 0.5$ indicates a synergistic interaction (EUCAST, 2000). The possible mechanisms underlying these interactions are described in a separate chapter.

\section{Synergy between plant-derived compounds and antibiotics or bacteriocins}

There have been a substantial number of reports on synergistic antibacterial activity between various purified plant-derived compounds and plant oils, and antibiotics (mainly $\beta$-lactams) against Staphylococcus aureus including (MRSA). The most relevant findings from these studies will be presented in chronological order. Brehm-Stecher and Johnson (2003) observed that treatment with low concentrations of the sesquiterpenoids nerolidol, bisabolol and apritone enhanced bacterial susceptibility to ciprofloxacin, clindamycin, erythromycin, gentamicin, tetracycline and vancomycin. Synergism was demonstrated between ampicillin and ethanolic extracts from 10 Indian medicinal plants, including Camelia sinensis (Chinese tea), that are rich in alkaloids, glycosides, flavanoids, phenols and saponins (Aqil et al., 2005), and quinic acid gallates from Caesalpina spinosa could intensify the susceptibility of MRSA to oxacillin (Kondo et al., 2006). Grande et al. (2007) found that the antimicrobial activity of bacteriocin produced by Enterococcus faecalis, enterocin AS-48, against $S$. aureus was potentiated when applied in combination with phenolic compounds such as carvacrol. Synergy was demonstrated between the diterpenoids salvipisone and aethiopinone, and antibiotics from the $\beta$-lactam, glycopeptide and oxazolidinone groups (Walencka et al., 2007). Nascimento et al. (2007) observed synergistic activity between ampicillin and the Brazilian plant Eremanthus erythropappus oil and $\beta$-bisakolene. The hop (Humulus lupulus)-derived compounds, lupulone and xanthohumol, showed synergy with tobramycin and ciprofloxacin (Natarajan et al., 2008). A synergistic effect of kaempferol glycosides 
Table I

Examples of synergy between novel antimicrobials and antibiotics/bacteriocins

\begin{tabular}{|c|c|c|c|}
\hline Novel antimicrobial & Antibiotic/bacteriocin & Bacterial species & Reference \\
\hline \multicolumn{4}{|l|}{ Plant compound } \\
\hline Thymol & Nisin & L. monocytogenes & Ettayebi et al., 2000 \\
\hline EGCg & Ampicillin + sulbactam & MRSA & Hu et al., 2001 \\
\hline EGCg & Penicillin & S. aureus & Zhao et al., 2002 \\
\hline Baicalein & $\beta$-lactams, tetracycline & MRSA & Fujita et al., 2005 \\
\hline 7-methyljuglone & Isoniazid & M. tuberculosis & Bapela et al., 2006 \\
\hline Carnosol & Aminoglycosides & VRE & Horiuchi et al., 2007 \\
\hline Asiatic acid, corosolic acid & Tobramycin & $P$. aeruginosa & Garo et al., 2007 \\
\hline Ellagic acid, tannic acid & Novobiocin & A. baumanii & Chusri et al., 2009 \\
\hline Kaempferol glycosides & Fluorochinolones & MRSA & Liu et al., 2009 \\
\hline Galangin & Cefazidime & PRSA & Eumkeb et al., 2010 \\
\hline Oleanolic acid & Rifampicin & M. tuberculosis & Ge et al., 2010 \\
\hline Betulic acid & Methicillin, vancomycin & S. aureus & Chung et al., 2011 \\
\hline Oleanolic acid, ursolic acid & Ampicillin, oxacillin & S. aureus, S. epidermidis & Kurek et al., 2012 \\
\hline \multicolumn{4}{|l|}{$\mathrm{Ag}^{+}$and AgNPs } \\
\hline $\mathrm{Ag}^{+}$ & Vancomycin, amoxicillin, penicillin G & S. aureus & Shahverdi et al., 2007 \\
\hline AgNPs & Polymyxin B & Gram $^{-}$bacteria & Ruden et al., 2009 \\
\hline AgNPs & Ampicillin & $\mathrm{Gram}^{+}$bacteria & Fayaz et al., 2010 \\
\hline \multicolumn{4}{|l|}{ Bacteriophages lytic enzymes } \\
\hline Cpl -1 & Cefotaxime, moxifloxacin & S. pneumoniae & Rogríguez-Cerrato et al., 2007 \\
\hline LysK & Lysostaphin & MRSA & Becker et al., 2008 \\
\hline ClyS & Oxacillin, vancomycin & MRSA & Daniel et al., 2010 \\
\hline LysH5 & Nizin & S. aureus & Garcia et al., 2010 \\
\hline
\end{tabular}

purified from Laurus nobilis and fluorochinolones on MRSA was shown by Liu et al. (2009), while the efficacy of galangin, a flavanol isolated from Alpinia officinarum, administered with ceftazidime, against penicillinresistant $\underline{S}$. a ureus (PRSA) was demonstrated by Eumkeb and coworkers (2010). In a study of the synergistic antimicrobial activity of pentacyclic triterpenoids (e.g. betulinic acid) combined with methicillin or vancomycin, it was found that various combinations of these compounds could reduce their minimal inhibitory concentrations (MICs) by $0.05-50 \%$ (Chung et al., 2011). Two other pentacyclic triterpenoids, oleanolic acid and ursolic acid, have recently been shown to act synergistically with ampicillin and oxacillin against $S$. aureus and Staphylococcus epidermidis grown in solution or as biofilms (Kurek et al., 2012).

In the last decade, there have been an appreciable number of reports describing synergy between plant compounds and antibiotics against bacteria outside the genus Staphylococcus. The antibacterial effect of nisin Z against Listeria monocytogenes ATCC 7644 and Bacillus subtilis ATCC 33712 was found to be greatly enhanced by a subinhibitory concentration of thymol (Ettayebi et al., 2000), and the diterpenoid carnosol reduced the MICs of various aminoglyco- sides against vancomycin-resistant enterococci - VRE (Horiuchi et al., 2007). Garo et al. (2007) showed that treatment with asiatic acid and corosolic acid enhanced the susceptibility of Pseudomonas aeruginosa bioilms to tobramycin. Alcoholic extracts from 15 traditional Indian medicinal plants exhibited synergy with tetracycline and ciprofloxacin to inhibit the growth of ESbetaL (extended spectrum beta-lactamase)-producing E. coli and Shigella (Ahmad and Aqil, 2007). The combination of kaempferol with clindamycin or quercetin produced a large synergistic effect against antibiotic-resistant Propionibacterium acnes (Lim et al., 2007). Studies by a Chinese group have confirmed the synergistic activity between a herbal medicine isolated from Ramulus cinnamoni and tetracycline, gentamicin and streptomycin against nosocomical antibiotic-resistant strains of P. aeruginosa (Liu et al., 2007). Using transmission electron microscopy, Sivarooban et al. (2008) observed cell damage in L. monocytogenes caused by a combination of nisin with either a grape seed or a green tea extract rich in phenolic constituents. Combination with gerianol isolated from Helichrysum italicum, a member of the sunflower family, significantly increased the efficacy of $\beta$-lactams, quinolones and chloramphenicol towards multidrug resistant 
Enterobacter aerogenes, E.coli, P. aeruginosa and Acinetobacter baumanii (Lorenzi et al., 2009). The antibacterial activity of novobiocin was shown to be enhanced by the plant phenolics ellagic and tannic acids, which increased its effectiveness against multi-drug resistant A. baumanii (Chusri et al., 2009). A crude leaf extract of Helichrysum pedunculatum enhanced the activity of eight antibiotics from various groups against bacteria implicated in wound infections (Aiyegoro et al., 2010). Mulyaningsih et al. (2010) elucidated the synergistic properties of two terpenoids from the essential oil of Eucalyptus globulus, aromadendrene and 1,8-cineole, against VRE. A notable study by Ge et al. (2010) described synergistic in vitro interactions between oleanolic acid and isoniazid, rifampicin or ethambutanol against Mycobacterium tuberculosis. The potent synergism of ciprofloxacin with extracts of medicinal plants, e.g. Angelica sinensis and Melissa officinalis, against Enterobacteriaceae and P. aeruginosa has also recently been reported (Garvey et al., 2011).

\section{Synergistic activity between metal ions or nanoparticles and antibiotics or bacteriocins}

The majority of reports on the interaction between antibiotics and various forms of metals describe the effects of silver ions and silver nanoparticles (AgNPs). In an early study, Modak and Fox (1985) identified synergism between silver sulfadazine and piperacillin (an extended-spectrum penicillin antibiotic), both in vitro and in vivo. The antimicrobial activities of various antibiotics in the presence of $\mathrm{Ag}^{+}$ions have since been studied more systematically and the greatest enhancing effect was observed for vancomycin, amoxicillin and penicillin G against S. aureus rather than E. coli (Shahverdi et al., 2007). In contrast, the synergistic activity between silver nanoparticles (AgNPs) and ampicillin, gentamicin, kanamycin, streptomycin and vancomycin was subsequently shown to be greater against E. coli and P. aeruginosa than against S. aureus (Birla et al., 2009). It was also confirmed that silver nanoparticles can enhance the antibacterial activity of chloramphenicol, being an active carrier of this antibiotic (Patil et al., 2009). It has yet to be determined whether nanoparticles pre-bound to an antibiotic produce a greater antimicrobial effect than simultaneous addition of silver and the antibiotic (Durán et al., 2010). A number of studies have shown that silver can act synergistically with compounds other than antibiotics. The activity of AgNPs against an E. coli biofilm was increased by the lipopeptide biosurfactant V9T14 (Rivardo et al., 2010), and synergy with chitosan against $S$. aureus has also been reported (Potara et al., 2011). Ammons et al. (2011) recently showed that a silver wound dressing combined with the immune molecule lactoferrin and the rare sugar-alcohol xylitol, reduced biofilm viability more effectively than standard silver hydrogel.

So far there have been no reports of the direct synergistic activity of gold nanoparticles (AuNPs) and antibiotics. Gu et al. (2003) found that AuNP-vancomycin conjugates can act as a potent inhibitor of VRE and E. coli. It has also been demonstrated that AuNPs function as useful carriers for ciprofloxacin and other fluoroquinolones (Tom etal., 2004). In the case of E. coli, the AuNP conjugate showed greater antibacterial activity than free ciprofloxacin (Rosemary et al., 2006). However, Burygin et al. (2009) found no difference between the antibacterial activity of a gentamicin conjugate with AuNPs and the free antibiotic. A single report has described the synergistic interaction between copper and an antibiotic - erythromycin (Sultana et al., 2005). Synergism was observed between this antibiotic and several other trace elements besides copper (e.g. cobalt, nickel, chromium), against both Gram-negative and Gram-positive bacteria. Synergistic antimicrobial effects between metal ions and compounds other than antibiotics have also been reported, the most well documented of which is enhancement of the antimicrobial activity of pomegranate extracts against clinical isolates of S.aureus and P. aeruginosa by combination with cupric sulfate (Gould et al., 2009 a, b).

\section{Antibiotics or bacteriocins and bacteriophage lytic enzymes}

There have been several recent reports describing interactions between bacteriophage-encoded lytic enzymes and classic antibiotics or bacteriocins. A synergistic effect with penicillin and gentamicin was observed for lytic enzyme Cpl-1 encoded by Streptococcus pneumoniae lytic phage Cp-1 and also for another endolysin, Pal, encoded by S. pneumoniae lytic phage Dp-1 (López and García, 2004), against several penicillin-resistant and -sensitive S. pneumoniae strains (Loeffler and Fischetti, 2003; Djurkovic et al., 2005). In vitro interactions between Cpl-1 and Pal with cefotaxime and moxifloxacin against antibiotic-susceptible and antibiotic-resistant $S$. pneumoniae have also been studied. Synergistic activity was confirmed for the combination of Cpl-1 and cefotaxime or moxifloxacin and the effect was strain-dependent. It is noteworthy that greater synergy was observed for the combination of these antibiotics with LytA, which is the major pneumococcal autolysin (Rodríguez-Cerrato et al., 2007). The combined effect of nisin and two S. aureus lytic phages, $\varphi 35$ and $\varphi 88$, was assessed, and a synergistic effect was observed in short-term experiments. However nisin adaptation and reciprocal resistance to 
both phages have prevented the practical application of this combined therapy (Martínez et al., 2008). Another two endolysins, LysK, produced by staphylococcal bacteriophage K (O'Flaherty et al., 2005) and the antistaphylococcal bacteriocin, lysostaphin, synthesized by Staphylococcus simulans (Dajcs et al., 2000), exhibited synergy in killing MRSA (Becker et al., 2008). Recently, a novel chimeric lysin, ClyS, was engineered by fusing the $\mathrm{N}$-terminal catalytic domain of S. aureus Twort phage lysin with the C-terminal cell-wall targeting domain of the phage $\varphi \mathrm{NM} 3$ lysin. The chimeric protein displayed synergistic interactions with both vancomycin and oxacillin in vitro and its combination with oxacillin could prevent septic death in MRSA-infected mice (Daniel et al., 2010).

\section{Molecular basis of synergistic activities}

Three categories of combination therapy can be distinguished (Fischbach, 2011). The most common strategy utilizes the combination of drugs which inhibit different pathways within bacterial cells. An example of such a strategy is treatment of Mycobacterium tuberculosis infections with four drugs: 1) isoniazid, an inhibitor of fatty acid synthesis, 2) rifampicin, an inhibitor of RNA polymerase, 3) ethambutanol, an inhibitor of arabinose transferases involved in cell wall biosynthesis, and 4) pyrozinamide, with an as yet unknown mechanism of action (Ginsberg and Spigelman, 2007). The second strategy is based on the inhibition of different targets in the same pathway. The inhibition of folic acid synthesis by a combination of sulfamethoxazole, an inhibitor of dihydropteroate synthetase, and trimethoprim, inhibiting dihydrofolate reductase, is based on this strategy (Wormster et al., 1982). The third strategy requires inhibition of the same target in different ways, e.g. the application of streptogramin and virginamycin, which both inhibit the peptidyl transferase center on the 50 S ribosomal subunit (Tu et al., 2005). It should be noted that such a combined antimicrobial effect is utilized in nature by antibiotic producers to compete effectively with other species (Ohnishi et al., 2008). Instead of two antibiotics, combination therapy can utilize antibiotics with their "sensitizers": molecules that make the co-applied antibiotic more effective by inhibiting enzymes responsible for antibiotic resistance or those that metabolize the drug. For example, diazabicyclooctanes (DBOs) are novel class A and class C $\beta$-lactamase inhibitors that are more potent than current commercially available inhibitors (Coleman, 2011). Similarly, the synergistic antibacterial activity between various plant-derived compounds increases the effectiveness of herbal extracts in comparison with the isolated single constituents. In phytotherapy, this synergy is more dif- ficult to dissect because plant extracts contain many minor agents that may influence the combined effect. In spite of this, many synergistic activities between phytopharmaceuticals have been demonstrated, and in some cases the mechanism of this effect has been elucidated (Wagner and Ulrich-Merzenich, 2009).

The synergistic antimicrobial effect of an antibiotic combined with another agent requires interaction of the latter compound with the bacterial resistance mechanism. The first details of the molecular basis of synergistic interaction between some plant-derived compounds and various classes of antibiotics have recently been revealed. The ability of novel therapeutics to inhibit lactam- or ester-cleaving enzymes can result in synergy with $\beta$-lactams. EGCg (epigallocatechin gallate) inhibits penicillinase activity, thus restoring the effectiveness of penicillin against S. aureus (Zhao et al., 2002) and potentiating the effect of ampicillin and sulbactam against MRSA (Hu et al., 2001). The synergy between galangin and methicillin, ampicillin, amoxicillin, cloxacillin, penicillin $G$ and cefazidime against $S$. aureus was found to be based on the marked inhibitory activity of galangin against penicillinase and $\beta$-lactamase (Eumkeb et al., 2010). The two antimicrobials in a combination may affect the same cellular target. For example, EGCg administered with a $\beta$-lactam antibiotic could inhibit peptidoglycan synthesis (Yam et al., 1998; Zhao et al., 2001). Subsequently, Fujita et al. (2005) demonstrated that the flavone baicalein exhibits remarkable synergy with $\beta$-lactam antibiotics against MRSA, possibly by inhibiting the activity of PBP $2 \mathrm{a}$ or by affecting peptidoglycan structure, and Kuroda et al. (2007) demonstrated that the sesquiterpene farnesol inhibits recycling of the $\mathrm{C}_{55}$ carrier of the murein monomer precursor, thus contributing to increased bacterial susceptibility to $\beta$-lactams. Several plant compounds appear to inhibit defined targets in the bacterial cell. The flavanol myricetin was found to suppress DnaB helicase activity and glycosylated flavones could inhibit topoisomerase IV, so these compounds have the potential to act synergistically with particular antibiotics (Hemaiswarya et al., 2008). Another mechanism of synergy is by increasing the intracellular antibiotic concentration, which may be achieved by overcoming cellular barriers that prevent antibiotics from penetrating the cell or by blocking bacterial efflux pumps that extrude such agents from the cell. The majority of reports have described the effect of plant compounds on bacterial efflux pumps. The indole alkaloid reserpine, a modulator of multidrug pumps enhanced tetracycline activity against MRSA containing the tet $K$ determinant (Gibbons and Udo, 2000), and baicalein inhibited TetK-dependent efflux of tetracycline (Fujita et al., 2005). Certain plant-derived compounds, e.g. EGCg, have been shown to act as bacterial efflux pump inhibitors (EPIs) and are able to restore 
the antibacterial effect of ineffective antibiotics such as ciprofloxacin, preferentially against Gram-positive, but also against Gram-negative species (Stavri et al., 2007). $\mathrm{N}$-caffeoylphenalkylamide derivatives were found to act as EPIs in S. aureus, especially in strains overexpressing the multidrug efflux transporter NorA (Michalet et al., 2007). Chusri et al. (2009) suggested that ellagic and tannic acids act as efflux pump inhibitors in A. baumanii. It was recently demonstrated that the aforementioned synergism between medicinal plant extracts and ciprofloxacin is the result of inhibition of the efflux pump in Gram-negative bacteria (Garvey et al., 2011). Another recent study showed that caffeoylquinic acids from Artemisia absinthium preferentially bind to Major Facilitator Super Family efflux systems, which are key multidrug resistance determinants in Gram-positive bacteria (Fiamegos et al., 2011). Plant-derived compounds may also be involved in the transformation of a non-active antimicrobial into its active form. The naphthoquinone 7-methyljuglone was able to potentiate the effect of antituberculous drugs against extracellular and intracellular Mycobacterium tuberculosis, possibly due to the elevated synthesis of superoxide, which catalyzes the transformation of isoniazid into its active form (Bapela et al., 2006). A recent attempt to elucidate the mechanism of synergy between oleanolic and ursolic acids and ampicillin failed to produce an unequivocal answer. However, the inactivation of ampicillin target, the PBPs (penicillin binding proteins), the inhibition of $\beta$-lactamase translocation and increased $\beta$-lactam transport mediated by these compounds, were all excluded (Kurek et al., 2012).

There have been few studies on the molecular basis of synergy between antibiotics and metal nanoparticles or bacteriophage lytic enzymes. It has been claimed that the synergism between nanoparticles and antibiotics or bacteriocins is based on the ability of the latter to help nanoparticles reach their cellular targets. Synergistic activity between silver nanoparticles and membranepermeabilizing antimicrobial peptides, such as the lipopeptide polymyxin B has been reported (Ruden et al., 2009). Polymyxin B is a cyclic polycationic lipopeptide that disrupts the outer membranes of Gram-negative bacteria by interacting with lipid A (Schindler and Osborn, 1979), and it was postulated that such antimicrobial peptides allow nanoparticles to gain access to their internal target site. Fayaz et al. (2010) showed that AgNPs can act synergistically with several antibiotics, preferentially against Gram-negative bacteria, and the greatest effect was observed with ampicillin. These authors proposed a model in which AgNPs associate with ampicillin, these complexes interact with the bacterial cell wall and subsequently inhibit the formation of peptidoglycan cross-links, leading to cell wall lysis. In addition, the AgNPs may prevent DNA unwinding when inside the cell. It has also been established that AuNPs are a very useful tool for drug delivery and serve as a stable and non-toxic platform for pharmaceuticals, enhancing their stability and improving targeting (Pissuwan et al., 2010).

The aforementioned synergy between two peptidoglycan hydrolases, endolysin LysK and lysostaphin, may be due to the fact that LysK has two lytic domain (endopeptidase and amidase) and thus is able to enhance the lytic potential of lysostaphin, which has single lytic domain (Becker et al., 2008). García et al. (2010) reported synergy between phage endolysin LysH5, which is active against a wide range of staphylococci (Obeso et al. 2008), and nisin in killing S. aureus in pasteurized milk. The MICs of nisin and LysH5 were diminished by 64- and 16-fold, respectively. It was postulated that LysH5 activity might be increased by the permeabilization of the cytoplasmic membrane by nisin, as was previously documented for the endolysin Lys44 (Nascimento et al., 2008).

\section{Conclusion}

The number and variety of novel antimicrobials which show synergy with classic antibiotics and bacteriocins is substantial. Many of these compounds have already been used as an alternative to conventional treatments in medicine and agriculture. However, their widespread application is restricted, mainly because their mechanisms of action have not been fully characterized and their effect on eukaryotes has yet to be established. The problem of antibiotic resistance among bacteria has received much coverage in the literature (Fernebro, 2011; Defoirdt et al., 2011). The proven synergistic activities of novel antimicrobials with well known antibiotics provides some hope that the latter may still be of use to treat diseases caused by antibiotic-resistant bacteria. Due to their narrow action spectrum and toxicity, bacteriocins were replaced by antibiotics in clinical use and are now extensively used in food preservation (Riley and Wertz, 2002; Falagas and Kasiakou, 2005). Bacteriocin-resistance may be countered by the use of these compounds in combination with novel antimicrobials. Such a strategy might restore the potential of bacteriocin (e.g. nisin) to eliminate pathogenic bacteria, like S. aureus, from food. Currently, novel antimicrobials cannot replace antibiotics, but they may become valuable antibiotic complements. In order to exploit these new antimicrobials effectively in synergistic combination therapy, it will be necessary to determine the optimal ratio and dosing regimen, and to fully characterize the mechanisms of their activities by employing genomic, proteomic and metabolomic technologies. 
Acknowledgment

This work was supported by Polish Ministry of Science and Higher Education grant NN 302027937.

\section{Literature}

Ahmad I. and F. Aqil. 2007. In vitro efficacy of bioactive extracts of 15 medicinal plants against ES beta L-producing multidrug resistant enteric bacteria. Microbiol. Res. 162: 264-275.

Aiyegoro O.A., A.J. Afolayan and A.I. Okoh. 2010. Interactions of antibiotics and extracts of Helichrysum pedunculatum against bacteria implicated in wound infections. Folia Microbiol. 55: 176-180. Aqil F., M.S. Khan, M. Owais and I. Ahmad. 2005. Effect of certain bioactive plant extracts on clinical isolates of beta-lactamase producing methicillin resistant Staphylococcus aureus. J. Basic Microbiol. 45: 106-114.

Ammons M.C., L.S. Ward and G.A. James. 2011. Anti-biofilm efficacy of a lactoferrin/xylitol wound hydrogel used in combination with silver wound dressings. Int. Wound J. 8: 268-273.

Bapela N.B., N. Lall, P.B. Fourie, S.G. Franzblau and C.E.J. van Rensburg. 2006. Activity of 7-methyljuglone in combination with antituberculous drugs against Mycobacterium tuberculosis. Phytomedicine 13: 630-635.

Becker S.C., J. Foster-Frey and D.M. Donovan. 2008. The phage K lytic enzyme LysK and lysostaphin act synergistically to kill MRSA. FEMS Microbiol. Lett. 287: 185-191.

Birla S.S., V.V. Tiwari, A.K. Gade, A.P. Ingle, A.P. Yadav and M.K. Rai. 2009. Fabrication of silver nanoparticles by Phoma glom erata and its combined effect agaist Escherichia coli, Pseudomonas aeruginosa and Staphylococcus aureus. Lett. Appl. Microbiol. 48: 173-179.

Brehm-Stecher B.F. and E.A. Johnson. 2003. Sensitization of Staphylococcus aureus and Escherichia coli to antibiotics by the sesquiterpenoids nerolidol, farnesol, bisabolol and apritone. Antimicrob. Agents Chemother. 47: 3357-3360.

Burygin G.L., B.N. Khlebtsov, A.N. Shantrokha, L.A. Dykman, V.A. Bogatyrev and N.G. Khlebtsov. 2009. On the enhanced antibacterial activity of antibiotics mixed with gold nanoparticles. Nanoscale Res. Lett. 4: 794-801.

Chibani-Chennoufi S., J. Sidoti, A. Bruttin, E. Kutter, S. Sarker and H. Brusow. 2004. In vitro and in vivo bacteriolytic activities of Escherichia coli phages: implications for phage therapy. Antimicrob. Agents Chemother. 48: 2558-2569.

Chopra I. 2007. The increasing use of silver-based products as antimicrobial agents: a useful development or a case for concern? J. Antimicrobial. Chem. 59: 587-590.

Chung P.Y., P. Navaratnam and L.Y. Chung. 2011. Synergistic antimicrobial activity between pentacyclic triterpenoids and antibiotics against Staphylococcus aureus strains. Ann. Clin. Microbiol. Animicrob. 10: 25-30.

Chusri S., I. Villanueva, S.P. Voravuthikunchai and J. Davies. 2009. Enhancing antibiotic activity: a strategy to control Acinetobacter infections. J. Antimicrob. Chemother. 64: 1203-1211.

Coleman K. 2011. Diazabicyclooctanes (DBOs): a potent new class of a non- $\beta$-lactam -lactamase inhibitors. Curr. Opin. Microbiol. 14: 550-555.

Cushnie T.P. and A.J. Lamb. 2011. Recent advances in understanding the antibacterial properties of flavonoids. Int. J. Antimicrob. Agents 38: 99-107.

Dajcs J.J., E.B. Hume, J.M. Moreau, A.R. Caballero, B.M. Cannon and R.J. O'Callaghan. 2000. Lysostaphin treatment of methicillin-resistant Staphylococcus aureus keratitis in the rabbit (1). Am. J. Ophtalmol. 130: 544.
Daniel A., C. Euler, M. Collin, P. Chahales, K.J. Gorelick and V.A. Fischetti. 2010. Synergism between a novel chimeric lysin and oxacillin protects against infection by methicillin-resistant Staphylococcus aureus. Antimicrob. Agents Chemother. 54: 1603-1612.

Defoirdt T., P. Sorgeloos and P. Bossier. 2011. Alternatives to antibiotics for the control of bacterial disease in aquaculture. Curr. Opin. Microbiol. 14: 251-258.

Djurkovic S., J.M. Loeffler and V.A. Fischetti. 2005. Synergistic killing of Streptococcus pneumoniae with the bacteriophage lytic enzyme Cpl-1 and penicillin or gentamicin depends on the level of penicillin resistance. Antimicrob Agents Chemother. 49: 1225-1228. Durán N., P.D. Marcato, R. De Conti, O.L. Alves, F.T.M. Costa and M. Brocchi. 2010. Potential use of silver nanoparticles on pathogenic bacteria, their toxicity and possible mechanisms of action. J. Braz. Chem. Soc. 21: 949-959.

EUCAST, 2000. Terminology relating to methods for the determination of susceptibility of bacteria to antimicrobial agents. http://www. escmid.org/Files/E_def_1_2_03_2000.pdf

Ettayebi K., J. El Yamani and B. Rossi-Hassani. 2000. Synergistic effect of nisin and thymol on antimicrobial activities in Listeria monocytogenes and Bacillus subtilis. FEMS Microbiol. Lett. 183: 191-195.

Eumkeb G., S. Sakdarat and S. Siriwong. 2010. Reversing $\beta$-lactam antibiotic resistance against Staphylococcus aureus with galangin from Alpinia officinarum Hance and synergism with ceftazidime. Phytomedicine 18: 40-45.

Falagas M.E. and S.K. Kasiakou. 2005. Colistin: the revival of polymyxins for the management of multidrug-resistant gram-negative bacterial infections. Clin. Infect. Dis. 40: 1333-1341.

Fayaz A.M., K. Balaji, M. Girilal, R. Yadav, P.T. Kalaichelvan and R. Venketesan. 2010. Biogenic synthesis of silver nanoparticles and their synergistic effect with antibiotics: a study against gram-positive and gram-negative bacteria. Nanomedicine 6: 103-109.

Fernebro J. 2011. Fighting bacterial infections - Future treatment options. Drug Resist. Updat. 14: 125-139.

Fiamegos Y.C., P.L. Kastritis, V. Exarchou, H. Han, A.M. Bonvin, J. Vervoort, K. Lewis, M.R. Hamblin and G.P. Tegos. 2011. Antimicrobial and efflux pump inhibitory activity of caffeoylquinic acids from Artemisia absinthium against Gram-positive pathogenic bacteria. PLoS ONE 6: e18127.

Fischbach M. 2011. Combination therapies for combating antibacterial resistance. Curr. Opin. Microbiol. 14: 519-523.

Fujita M., S. Shiota, T. Kuroda, T. Hatano, T. Yoshida, T. Mizushima and T. Tsuchiya. 2005. Remarkable synergies between baicalein and tetracycline and baicalein and $\beta$-lactam against methicillinesistant Staphylococcus aureus. Microbiol. Immunol. 49: 391-396.

García P., B. Martínez, L. Rodríguez and A. Rodríguez. 2010. Synergy between the phage endolysin LysH5 and nisin to kill Staphylococcus aureus in pasteurized milk. Int. J. Food Microbiol. 141: 151-155.

Garo E., G.R. Eldridge, M.G. Goering, E. DeLancey Pulcini, M.A. Hamilton, J.W. Costerton and G.A. James. 2007. Asiatic acid and corosolic acid enhance the susceptibility of Pseudomonas aeruginosa biofilms to tobramycin. Antimicrob. Agents Chemother. 51: 1813-1817.

Garvey M.I., M.M. Rahman, S. Gibbons and L.J. Piddock. 2011. Medical plant extracts with efflux inhibitory activity against Gramnegative bacteria. Int. J. Antimicrob. Agents 37: 145-151.

Ge F., F. Zeng, S. Liu, N. Guo, H. Ye, Y. Song, J. Fan, X. Wu, X. Wang, X. Deng, Q. Jin and L. Yu. 2010. In vitro synergistic interactions of oleanolic acid in combination with isoniazid, rifampicin or ethambutanol against Mycobacterium tuberculosis. J. Med. Microbiol. 59: 567-572.

Gibbons S. and E.E. Udo. 2000. The effect of reserpine, a modulator of multidrug pumps, on the in vitro activity of tetracycline against 
clinical isolates of methicillin resistant Staphylococcus aureus (MRSA) possessing the tet $(k)$ determinant. Phytother. Res. 14: 139-140. Ginsberg A.M. and M. Spigelman. 2007. Challenges in tuberculosis drug research and development. Nat. Med. 13: 290-294.

Gould S.W., M.D. Fielder, A.F. Kelly and D.P. Naughton. 2009a. Anti-microbial activities of pomegranate rind extracts: enhancement by cupric sulphate against clinical isolates of $S$. aureus, MRSA and PVL positive CA-MSSA. BMC Complement. Altern. Med. 9: 23 Gould S.W., M.D. Fielder, A.F. Kelly, W. El Sankary and D.P. Naughton. 2009 b. Antimicrobial pomegranate rind extracts: enhancement by $\mathrm{Cu}(\mathrm{II})$ and vitamin $\mathrm{C}$ combinations against clinical isolates of Pseudomonas aeruginosa. Br. J. Biomed. Sci. 66: 129-132. Górski A., A. Międzobrodzki, J. Weber-Dabrowska, M. Łobocka, W. Fortuna, S. Letkiewicz, M. Zimecki and G. Filby. 2009. Bacteriophage therapy for the treatment of infections. Curr. Opin. Investig. Drugs 10: 766-774.

Grande M.J., R.L. López, H. Abriouel, E. Valdivia, N. Ben Omar, M. Maqueda, M. Martinez-Caňamero and A. Gálvez. 2007. Treatment of vegetable sauce with enterocin AS-48 alone or in combination with phenolic compounds to inhibit proliferation of Staphylococcus aureus. J. Food Prot. 70: 405-411.

Grandgirard D., J.M. Loeffler, V.A. Fischetti and S.I. Lieb. 2008. Phage lytic enzyme CpL-1 for antibacterial therapy in experimental pneumococcal meningitis. J. Infect. Dis. 197: 1519-1522.

Grudniak A.M., A. Kurek, J. Szarlak and K.I. Wolska. 2011. Oleanolic and ursolic acids influence the expression of the cysteine regulon and the stress response in Escherichia coli. Curr. Microbiol. 62: 1331-1336.

Gu H., P.L. Ho, E. Tong, L. Wang and B. Xu. 2003. Presenting vancomycin on nanoparticles to enhance antimicrobial activities. Nano Lett. 3: 1261-1263.

Hamill T.M., B.F. Gilmore, D.S. Jones and S.P. Gorman. 2007. Strategies for the development of the urinary catheters. Expert Rev. Med. Devices 4: 215-225.

Hardes J., C. von Eiff, A. Streitbuerger, M. Balke, T. Budny, M.P. Henrichs, G. Hauschild and H. Ahrens. 2010. Reduction of periprosthetic infection with silver-coated megaprostheses in patients with bone sarcoma. J. Surg. Oncol. 101: 389-395.

Hemaiswarya S., A.K. Kruthiventi and M. Doble. 2008. Synergism between natural products and antibiotics against infectious diseases. Phytomedicine 15: 639-652.

Horiuchi K., S. Shiota, T. Kuroda, T. Hatano, T. Yoshida and T. Tsuchiya. 2007. Potentiation of antimicrobial activity of aminoglycosides by carnosol from Salvia officinalis. Biol. Pharm. Bull. 30: 287-290.

Hu Z.-Q., W.-H. Zhao, Y. Hara and T. Shimamura. 2001. Epigallocatechin gallate synergy with ampicillin/sulbactam against 28 clinical isolates of methicillin-resistant Staphylococcus aureus J. Antimicrob. Chemother. 48: 361-364.

Im A.R., L. Han, E.R. Kim, Y.S. Kim and Y. Park. 2011. Enhanced antibacterial activities of Leonuri Herba extracts containing silver nanoparticles. Phytother. Res. Doi: 10.1002/ptr.3683.

Kaneko M., N. Togashi, H. Hamashima, M. Hirohara and Y. Inoue. 2011. Effect of farnesol on mevalonate pathway of Staphylococcus aureus. J. Antibiot. 64: 547-549.

Kondo K., Y. Takaishi, H. Shibata and T. Higuti. 2006. ILSMRs (intensifier of beta-lactam-susceptibility in methicillin-resistant Staphylococcus aureus) from Tara [Caesalpinia spinosa (Molina) Kuntze]. Phytomedicine 13: 209-212.

Kozai K., J. Suzuki, M. Okada and N. Nagasaka. 1999. Effect of oleanolic acid-cyclodextrin inclusion compounds on dental carries by in vitro experiment in rat-carries model. Microbios 97: 179-188. Kurek A., A.M., Grudniak, M. Szwed, A. Klicka, Ł. Samluk and K.I. Wolska. 2010. Oleanolic acid and ursolic acid affect peptido- glycan metabolism in Listeria monocytogenes. Anton. Leuven. Int. J. Mol. Biol. 97: 61-68.

Kurek A., A.M. Grudniak, A. Kraczkiewicz-Dowjat and K.I. Wolska. 2011. New antibacterial therapeutics and strategies. Pol. J. Microbiol. 60: 3-12.

Kurek A., P. Nadkowska, S. Pliszka and K.I. Wolska. 2012. Modulation of antibiotic resistance in bacterial pathogens by oleanolic acid and ursolic acid. Phytomedicine, doi: 10.1016/j. phymed. 2011.12.009.

Kuroda M., S. Nagasaki and T. Ohta. 2007. Sesquiterpene farnesol inhibits recycling of the $\mathrm{C}_{55}$ lipid carrier of the murein monomer precursor contributing to increased susceptibility to $\beta$-lactams in methicillin-resistant Staphylococcus aureus. J. Antimicrob. Chemother. 59: 425-432.

Li Y., P. Leung, Q.W. Song and E. Newton. 2006. Antimicrobial effect of surgical masks coated with nanoparticles. J. Hosp. Infect. 62: 58-63.

Lim Y.H., I.H. Kim and J.J. Seo. 2007. In vitro activity of kaempferol isolated from Impatiens balsamina alone and in combination with erythromycin and clindamycin against Propionnibacterium acnes. J. Microbiol. 45: 473-477.

Lin J.J., W.C. Lin, R.X. Dong and S.H. Hsu. 2012. The cellular responses and antibacterial activities of silver nanoparticles stabilized by different polymers. Nanotechnology 23: 065102.

Liu C.S., T.M. Cham, C.H. Yang, H.W. Chang, C.H. Chen and L.Y. Chuang. 2007. Antibacterial properties of Chinese herbal medicines against nosocomical antibiotic resistant strains of Psuedomonas aeruginosa in Taiwan. Am. J. Chin. Med. 35: 1047-1060.

Liu M.H., N. Otsuka, K. Noyori, S. Shiota, W. Ogawa, T. Kuroda, T. Hatano and T. Tsuchiya. 2009. Synergistic effect of kaempferol glycosides purified from Laurus nobilis and fluoroquinolones on methicillin-resistant Staphylococcus aureus. Biol. Pharm. Bull. 32: 489-492.

Loeffler J.M. and V.A. Fischetti. 2003. Synergistic lethal effect of a combination of phage lytic enzymes with different activities on penicillin-sensitive and -resistant Streptococcus pneumoniae strains. Antimicrob. Agents Chemother. 47: 375-377.

Lok C.M., C.M. Ho, R. Chen, Q.Y. He, W-Y. Yu, H. Sun, P.K. Tam, J.F. Chiu and C.M. Che. 2006. Proteomic analysis of the mode of antibacterial action of silver nanoparticles. J. Prot. Res. 5: 916-924.

López R. and E. García. 2004. Recent trends on the molecular biology of pneumococcal capsules, lytic enzymes and bacteriophage. FEMS Microbiol. Rev. 28: 553-580.

Lorenzi V., A. Muselli, A.F. Bernardini, L. Berti, J.M. Pages, L. Amaral and J.M. Bolla. 2009. Geraniol restores antibiotic activities against multidrug-resistant isolates from gram-negative species. Antimicrob. Agents Chemother. 53: 2209-2211.

Martínez B., J.M. Obeso, A. Rodríguez and P. García. 2008. Nisinbacteriophage crossresistance in Staphylococcus aureus. Int. J. Food Microbiol. 122: 253-258.

Michalet S., G. Cartier, B. David, A.M. Mariotte, M.G. DijouxFranca, G.W. Kaatz, M. Stavri and S. Gibbons. 2007. N-caffeoylphenalkylamide derivatives as bacterial efflux pump inhibitors. Bioorg. Med. Chem. Lett. 17: 1755-1758.

Modak S. and C.L. Fox Jr. 1985. Synergistic action of silver sulfadiazine and sodium piperacillin on resistant Pseudomonas aeruginosa in vitro and in experimental burn wound infections. J. Trauma. 25: 27-31.

Monteiro D.R., L.F. Gorup, A.S. Takamiya, A.C. Ruvollo-Filho, E.R. de Camargo and D.B. Barbosa. 2009. The growing importance of materials that prevent microbial adhesion; antimicrobial effect of metal devices containing silver. Int. J. Antimicrob. Agents 34: 103-110. 
Mulyaningsih S., F. Sporer, S. Zimmermann, J. Reichling and M. Wink. 2010. Synergistic properties of the terpenoids aromadendrene and 1,8-cineole from the essential oil of Eucalyptus globulus against antibiotic-susceptible and antibiotic-resistant pathogens. Phytomedicine 17: 1061-1066.

Nascimento A.M., M.G. Brandăo, G.B. Oliveira, I.C. Fortes and E. Chartone-Souza. 2007. Synergistic bactericidal activity of Eremanthus erythropappus oil or $\beta$-bisabolene with ampicillin against Staphylococcus aureus. Anton. Loeven. Int J. Mol. Biol. 92: 95-100.

Nascimento J.G., M.D. Guerreiro-Pereira, S.F. Costa, C. SăoJosé and M.A. Santos. 2008. Nisin-triggered activity of Lys44, the secreted endolysin from Oenococcus oeni phage fOg44. J. Bacteriol. 190: 457-461.

Natarajan P., S. Katta, I. Andrei, V. Babu Rao Ambati, M. Leonida, G.J. Haas. 2008. Positive antibacterial co-action between hop (Humulus lupulus) constituents and selected antibiotics. Phytomedicine 15: 194-201.

Neal A.I. 2008. What can be inferred from bacterium - nanoparticle interactions about the potential consequences of environmental exposure to nanoparticles? Exotoxicobiology 7: 362-371.

Nelson D., L. Loomis and V.A. Fischetti. 2001. Prevention and elimination of upper respiratory colonization of mice by group A streptococci by using a bacteriophage lytic enzyme. Proc. Natl. Acad. Sci. U.S.A. 98: 4107-4112.

Obeso J.M., B. Martínez, A. Rodríguez and P. García. 2008. Lytic activity of the recombinant staphylococcal bacteriophage phiH5 endolysin active against Staphylococcus aureus in milk. Int. J. Food Microbiol. 128: 212-218.

O’Flaherty S., A. Coffey, W. Meaney, G.F. Fitzgerald and R.P. Ross. 2005. The recombinant phage lysin LysK has a broad spectrum of lytic activity against clinically relevant staphylococci, including methicillin-resistant Staphylococcus aureus. J. Bacteriol. 187: 7161-7164.

Ohnishi Y., J. Ishikawa, H. Hara, H. Suzuki, M. Ikenoya, H. Ikeda, A. Yamashita, M. Hattori and S. Horinouchi. 2008. Genome sequence of the streptomycin-producing microorganism Streptomyces griseus IFO 13350. J. Bacteriol. 190: 4050-4060.

Parson J.B. and C.O. Rock. 2011. Is bacterial fatty acid synthesis a valid target for antibacterial drug discovery? Curr. Opin. Microbiol. 14: 544-549.

Patil S.S., R.S. Dhumal, M.V. Varghese, A.R. Paradkar and P.K. Khanna. 2009. Synthesis and antibacterial studies of chloramphenicol loaded nano-silver against Salmonella typhi. Synth. React. Inorg. Met-Org. Nano-Met. Chem. 39: 65-72.

Pissuwan D., C.H. Cortie, S.M. Valenzuela and M.B. Cortie. 2010. Functionalised gold nanoparticles for controlling patogenic bacteria. Trends Biotechnol. 28: 207-213.

Plaper A., M. Golob, I. Hafner, M. Oblak, T. Solmajer and R. Jerala. 2003. Characterization of quercetin binding site on DNA gyrase. Biochem. Biophys. Res. Commun. 306: 530-536.

Potara M., E. Jakab, A. Damert, O. Popescu, V. Canpean and S. Astilean. 2011. Synergistic antibacterial activity of chitosansilver nanocomposites on Staphylococcus aureus. Nanotechnology 22: 135101.

Rai M., A. Yadav and A. Gade. 2009. Silver nanoparticles as a new generation of antimicrobials. Biotech. Adv. 27: 76-83.

Rashel M., J. Uchiyama, T. Ujihara, Y. Uehara, S. Kuramoto, S. Sugihara, K. Yagyu, A. Muraoka, M. Sugai, K. Hiramatsu, K. Honke and S. Matsuzaki. 2007. Efficient elimination of multidrug-resistant Staphylococcus aureus by cloned lysine derived from bacteriophage phi MR11. J. Infect. Dis. 196: 1237-1247.

Ren D., R. Zuo, A.F. Gonzalez Barrios, L.A. Bedzyk, G.R. Eldridge, M.E. Pasmore and T.K. Wood. 2005. Differential gene expression for investigation of Escherichia coli biofilm inhibition by plant extract ursolic acid. Appl. Environ. Microbiol. 71: 4022-4034.

Riley M.A. and J.E. Wertz. 2002. Bacteriocins: evolutions, ecology, and application. Annu Rev. Microbiol. 56: 117-137.

Rivardo F., M.G. Martinotti, R.J. Turner and H. Ceri. 2010. The activity of silver against Escherichia coli biofilm is increased by a lipopeptide biosurfactant. Can. J. Microbiol. 56: 272-278.

Rodríguez-Cerrato V., P. García, G. del Prado, E. García, M. Garcia, L. Huelves, C. Ponte, R. López and F. Soriano. 2007. In vitro interactions of LytA, the major pneumococcal autolysin, with two bacteriophage lytic enzymes (Clp-1 and Pal), cefotaxime and moxifloxacin against antibiotic-susceptible and -resistant Streptococcus pneumoniae strains. J. Antimicrob. Chemother. 60: 1159-1162. Rosemary M.J., I. McLaren, T. Pradeep. 2006. Investigations of the antibacterial properties of ciprofloxacin $\mathrm{SiO}_{2}$. Langmuir. 22: 10125-10129.

Ruden S., K. Hilpert, M. Berditsch, P. Wadhwani and A.S. Ulrich. 2009. Synergistic interaction between silver nanoparticles and membrane-permeabilizing antimicrobial peptides. Antimicrob. Agents Chemother. 53: 3538-3540.

Schindler M. and M.J. Osborn. 1979. Interaction of divalent cations and polymyxin B with lipopolysaccharide. Biochemistry 18: 4425-4430.

Shahverdi A.R., A. Fakhimi, H.R. Shahverdi and S. Minaian. 2007. Synthesis and effect of silver nanoparticles on the antibacterial activity of different antibiotics against Staphylococcus aureus and Escherichia coli. Nanomedicine 3: 168-171.

Singh M., S. Singh, S. Prasada and I.S. Gambhir. 2008. Nanotechnology in medicine and antibacterial effect of silver nanoparticles. Digest J. Nanomat. Biostruct. 3: 115-122.

Sivarooban T., N.S. Hettiarachchy and M.G. Johnson. 2008. Transmission electron microscopy study of Listeria monocytogenes treated with nisin in combination with either grape seed or green tea seed. J. Food Prot. 71: 2105-2109.

Srivastava A., M. Talaue, S. Liu, D. Degen, R.Y. Ebright, E. Sineva, A. Chakraborty, S.Y. Druzhinin, S. Chatterjee, J. Mukhopadhyay, J.W. Ebright, A. Zozula, J. Shen, S. Sengupta, R.R. Niedfeldt, C. Xin, T. Kaneko, H. Irschik, R. Jansen, S. Donadio, N. Connell and R.H. Ebright 2011. New target for inhibition of bacterial RNA polymerase: "swith region". Curr. Opin. Microbiol. 14: 532-543.

Stavri M., L.J. Piddock and S. Gibbons. 2007. Bacterial efflux pump inhibitors from natural sources. J. Antimicrob. Chemother. 59: $1247-1260$.

Sultana N., M.S. Arayne and R. Sabri. 2005. Erytromycin synergism with essential and trace elements. Pak. J. Pharm. Sci. 18: 35-39. Theuretzbacher U. 2011. Resistance drives antibacterial drug development. Curr. Opin. Pharmacol. 11: 433-438.

Tom R.T., V. Suryanarayanan, P.G. Reddy, S. Baskaran and T. Pradeep. 2004. Ciprofloxacin-protected gold nanoparticles. Langmuir. 20: 1909-1914.

Tsuchiya H. and M. Iinuma. 2000. Reduction of membrane fluidity by antibacterial sophoraflavanone G isolated from Sophora exigua. Phytomedicine 7: 161-165.

Tu D., G. Blaha, P.B. Moore and T.A. Steitz. 2005. Structures of MLSBK antibiotics bound to mutated large ribosomal subunits provide a structural explanation of resistance. Cell 121: 257-270.

Vitiello M., M. Galdiero, E. Finamore, S. Galdiero and M. Galdiero. 2012. NF- $\mathrm{\kappa B}$ as a potential therapeutic target in microbial diseases. Mol. Biosyst. 8: 1108-1120.

Wagner H. and G. Ulrich-Merzenich. 2009. Synergy research: approaching a new generation of phytopharmaceuticals. Phytomedicine 2-3: 97-110.

Walencka E., S. Różalska, H. Wysokińska, M. Różalski, L. Kuźma and B. Różalska. 2007. Salvipisone and aethopinone from Salvia 
sclarea hairy roots modulate staphylococcal antibiotic resistance and express anti-biofilm activity. Planta Med. 73: 545-551.

Wolska K.I., A.M. Grudniak, B. Fiecek, A. Kraczkiewicz-Dowjat, A. Kurek. 2010. Antibacterial activity of oleanolic and ursolic acids and their derivatives. Centr. Eur. J. Biol. 5: 543-553.

Wormser G.P., G.T. Keusch and R.C. Heel. 1982. Co-trimoxazole (trimethoprim-sulfamethoxazole): an updated review of its antibacterial activity and clinical efficacy. Drugs 24: 459-518.

Yam Y.S., J.M.T. Hamilton-Miller and S. Shah. 1998. The effect of a component of tea (Camellia sinensis) on methicillin resistance, PBP2' synthesis, and $\beta$-lactamase production in Staphylococcus aureus. J. Antimicrob. Chemother. 42: 211-216.
Yoong P., R. Schuch, D. Nelson and V.A. Fischetti. 2006. PlyPH, a bacteriolytic enzyme with a broad $\mathrm{pH}$ range of activity and lytic action against Bacillus anthracis. J. Bacteriol. 188: 2711-2714.

Zhao W.-H., Z.Q. Hu, S. Okuba, Y. Hara and T. Shimamaura. 2001. Mechanism of synergy between epigallocatechin gallate and $\beta$-lactams against methicillin-resistant Staphylococcus aureus. Antimicrob. Agents Chemother. 45: 1737-1742.

Zhao W.-H., Z.-. Hu, Y. Hara, T. Shimamura. 2002. Inhibition of penicillinase by epigallocatechin gallate resulting in restoration of antibacterial activity of penicillin against penicillinase producing Staphylococcus aureus. Antimicrob. Agents Chemother. 36: 2266-2268. 